\title{
Higher expression of A-kinase anchoring-protein 1 predicts poor prognosis in human hepatocellular carcinoma
}

\author{
JIAN YU $^{1 *}$, YU ZHANG ${ }^{2 *}$, DONGXUN ZHOU ${ }^{3 *}, J_{N N} W^{3}$ and RONG LUO ${ }^{1}$ \\ ${ }^{1}$ Department of Endocrinology, CHC International Hospital, Cixi, Zhejiang 315315; ${ }^{2}$ Department of Oncology, Xiangyang \\ Central Hospital, Affiliated Hospital of Hubei University of Arts and Science, Xiangyang, Hubei 441000; \\ ${ }^{3}$ Department of Endoscopy, Eastern Hepato-Biliary Surgery Hospital, Shanghai 200438, P.R. China
}

Received September 11, 2017; Accepted February 19, 2018

DOI: $10.3892 / \mathrm{ol} .2018 .8685$

\begin{abstract}
A-kinase anchoring protein 1 (AKAP1) plays important regulatory roles in the regulation of mitochondrial function, oxidative metabolism, and cell survival. However, the expression pattern and prognostic value of AKAP1 in hepatocellular carcinoma (HCC) remains unclear. AKAP1 expression levels in tumor and matched non-tumor tissues were evaluated using reverse transcription-quantitative polymerase chain reaction and immunohistochemical staining. Kaplan-Meier and Cox regression analyses were used to analyze the survival rates. We found that AKAP1 protein expression was increased in HCC tissues, and high AKAP1 expression was associated with tumor size $(\mathrm{P}=0.024)$, Tumor-Node-Metastasis stage $(\mathrm{P}=0.0296)$ and portal vein thrombosis $(\mathrm{P}=0.00498)$. Kaplan-Meier survival analyses further revealed that high AKAP1 expression was associated with poor overall $(\mathrm{P}=0.004)$ and disease-free survival (DFS) $(\mathrm{P}=0.002)$ rates in patients with $\mathrm{HCC}$. Multivariate survival analysis revealed that AKAP1 served as an independent poor prognostic factor for DFS rates. The findings of the present study indicated that AKAP1 expression may contribute to HCC progression. High AKAP1 expression could serve as a valuable prognostic biomarker in predicting the survival of patients with HCC following radical resection.
\end{abstract}

\section{Introduction}

As the main histological subtype of liver cancer, hepatocellular carcinoma (HCC) is the third-leading cause of cancer-associated mortality worldwide $(1,2)$. There are $>700,000$ incidences of morality due to HCC annually $(3,4)$. China in particular

Correspondence to: Dr Rong Luo, Department of Endocrinology, CHC International Hospital, 599 Shiji Avenue, Guanhaiwei, Cixi, Zhejiang 315315, P.R. China

E-mail: 13586601688@163.com

${ }^{*}$ Contributed equally

Key words: A-kinase anchoring protein 1, hepatocellular carcinoma, prognosis has a high incidence rate of HCC, accounting for over half of new cases and mortalities, with $>422,100$ HCC-associated mortalities in $2015(5,6)$. Extensive studies have shown that the long-term prognosis of HCC remains dismal following radical excision owing to the high frequencies of tumor recurrence and distant metastases (6). Therefore, it is necessary to identify novel prognostic factors to guide clinical disease management following surgical resection.

A-kinase anchoring protein 1 (AKAP1, also known as AKAP121 and AKAP149) is a scaffold protein that integrates protein kinase A (PKA) and other signaling components to the outer mitochondrial membrane (7). Recent studies have revealed the roles of AKAP1 in regulating mitochondrial function, oxidative metabolism and cell survival $(8,9)$. AKAP1 recruits the PKA holoenzyme to specific subcellular sites and substrates, which is critical for the physiological actions of the kinase (10). AKAP1 also interacts with protein-tyrosine phosphatase D1 (PTPD1), a non-receptor tyrosine phosphatase that activates Src tyrosine kinase by binding with it (11).

In the heart, AKAP1 deficiency promotes cardiac mitochondrial aberrations and mitophagy, which enhances infarct size, pathological cardiac remodeling and increases the mortality rate from ischemic conditions (9). A more recent study (12) also demonstrated that AKAP1 is a transcriptional target of Myc and supports the growth of cancer cells. Upregulation of AKAP1 in high-grade human tumors is associated with enhanced mechanistic target of rapamycin (mTOR) activation and reduced patient survival. Knockdown of AKAP1 inhibited the mTOR pathway and impaired glioblastoma growth. However, the expression pattern and significance of AKAP1 in $\mathrm{HCC}$ remains to be investigated.

Despite the growing evidence of a link between AKAP1 and cancer, little is known about the expression level of AKAP1 and its clinicopathological significance in human HCC. The present study evaluated AKAP1 expression in paired tumorous and non-tumorous HCC samples. Next, the prognostic value of AKAP1 was determined in patients with HCC who previously underwent hepatectomies.

\section{Patients and methods}

Patients and samples. Formalin-fixed, paraffin-embedded HCC tissues from 141 male and 17 female patients (age range, 
18 to 78 years; median age, 50 years) who underwent curative resection at Eastern Hepatobiliary Surgery Hospital (Shanghai, China) between September 2006 and July 2011 were randomly retrieved. Another 30 pairs of fresh HCC tissues and the corresponding adjacent non-tumorous tissues (peritumoral tissues) were collected (26 male and 4 female patients; age range, 32 to 72 years; median age, 51 years) for reverse transcription-quantitative polymerase chain reaction (RT-qPCR). All human sample collection procedures were approved by the Biomedical Ethics Committee of Eastern Hepatobiliary Surgery Hospital (Shanghai, China). Informed consent for tissue banking and future medical research was obtained from each participant prior to surgery. The diagnosis of $\mathrm{HCC}$ was confirmed by pathological tests in all patients. The clinical staging was determined by the Tumor-Node-Metastasis (TNM) classification system (13). All patients were followed up until December 2014. The overall survival (OS) time was defined as the interval between the dates of surgery and death, and the disease-free survival (DFS) time was defined as the interval between the dates of surgery and first incidence of recurrence. If recurrence was not diagnosed, patients were censored at the date of mortality or the last follow-up.

Public database. A publically available cohort of human HCC patients from the National Center for Biotechnology Information Gene Expression Omnibus (GEO) dataset GSE45436 (https://www.ncbi.nlm.nih.gov/geo/query/acc. cgi?acc $=$ GSE45436) was used. This dataset included data from 39 samples of normal liver tissues and 95 samples of HCC tissues.

$R T-q P C R$. Total RNA was extracted from the fresh samples using TRIzol reagent (Invitrogen; Thermo Fisher Scientific, Inc., Waltham, MA, USA), following manufacturer instructions. RNA was then reversed transcribed to cDNA with Superscript III RT (Invitrogen; Thermo Fisher Scientific, Inc.) and random primers. Levels of AKAP1 and $18 \mathrm{~S}$ were measured by SYBR Green qPCR Master Mix (Takara Bio, Inc., Otsu, Japan) using an ABI PRISM7300HT Sequence Detection system (Applied Biosystems; Thermo Fisher Scientific, Inc.). The 18S rRNA expression level in the corresponding tissue was used as an internal control. Primers were designed as follows: AKAP1 forward, 5'-GCTTACGGCTTGTACCTG AAG-3' and reverse, 5'-ATGGTGCTCTTGGAAATACGC-3'; and $18 \mathrm{~S}$ forward, 5'-CGGCTACCACATCCAAGGAA-3' and reverse, 5'-GCTGGAATTACCGCGGCT-3'. The PCR reactions were performed under the following conditions: Initial denaturation at $95^{\circ} \mathrm{C}$ for $15 \mathrm{~min}$, followed by 40 cycles of $95^{\circ} \mathrm{C}$ for $30 \mathrm{sec}, 60^{\circ} \mathrm{C}$ for $20 \mathrm{sec}$ and $72^{\circ} \mathrm{C}$ for $30 \mathrm{sec}$. All samples were run in triplicate, and the data were normalized to the $18 \mathrm{~S}$ internal controls. The relative AKAP1 expression levels were calculated based on the $2^{-\Delta \Delta C q}$ method (14).

Histopathological and immunohistochemical evaluation. The formalin-fixed paraffin-embedded HCC tissue blocks were cut into $5 \mu \mathrm{m}$-thick sections. Then sections were deparaffinized at room temperature by $100 \%$ xylene ( 2 times, 5 min each) and rehydrated using a graded alcohol series ( 2 changes of $100 \%$ ethanol for $5 \mathrm{~min}$ each; 95 and $70 \%$ ethanol for 5 min each). Following incubation with $3 \%$ hydrogen peroxide for $20 \mathrm{~min}$ at room temperature, to quench endogenous peroxidase activity, the sections were processed for heat-induced antigen retrieval in sodium citrate buffer $(\mathrm{pH}=6.0)$. After several washes with $0.01 \mathrm{M}$ phosphate buffer, the sections were incubated with $10 \%$ goat serum (Beyotime Institute of Biotechnology, Shanghai, China) for $30 \mathrm{~min}$ at room temperature to block non-specific binding. Next, the slides were incubated with primary anti-AKAP1 antibody (cat no. 5203; dilution, 1:200; Cell Signaling Technology, Inc., Danvers, MA, USA) overnight at $4^{\circ} \mathrm{C}$. After the primary antibody was washed off, anti-rabbit peroxidase-conjugated secondary antibody (cat no. sc-2357; dilution, 1:500; Santa Cruz Biotechnology, Inc., Dallas, TX, USA) incubation was performed for $30 \mathrm{~min}$ at room temperature, and then DAB reagent (Dako; Agilent Technologies, Inc., Santa Clara, CA, USA) was utilized for detection. The staining of slides was observed and images captured using an Olympus microscope (IX-70 Olympus Corporation, Tokyo, Japan). All sections were evaluated by two independent pathologists who were blind to the clinicopathological data of the patients.

The percentages of positive tumor cells were semi-quantitatively graded as follows: $0,<5 \% ; 1,5-25 \% ; 2,26-50 \%$; and $3,>50 \%$. The staining intensity of tumor cells was scored as follows: 0 , negative staining; 1 , weak staining; 2 , moderate staining; and 3 , strong staining. The two scores were multiplied to obtain the final immunoreactive score. High expression of AKAP1 in tumor cells was defined as an immunoreactive score $\geq 4$.

Statistical analysis. Each experiment was performed in triplicate and data are presented as the mean \pm standard deviation. SPSS version 16 (SPSS, Inc., Chicago, IL, USA) was used for statistical analysis. $\chi^{2}$ tests were applied to determine statistical significance. Kaplan-Meier and Cox regression analyses were used to perform survival analysis. $\mathrm{P}<0.05$ was considered to indicate a statistically significant difference.

\section{Results}

Increased expression of AKAPI in patients with HCC. To determine the expression of AKAP1 in patients with HCC, RT-qPCR assays were performed for 30 pairs of HCC tissues and the corresponding adjacent non-tumorous tissues (peritumoral tissues). As shown in Fig. 1A, AKAP1 mRNA levels were significantly higher in HCC tissues. Consistently, analysis of public microarray data (GEO accession number GSE45436) also revealed that AKAP1 is significantly upregulated in tumor samples (Fig. 1B). To investigate AKAP1 expression in HCC tissues further, its expression was assessed in 158 cases of HCC and matched adjacent tissues by immunohistochemical (IHC) staining. As shown in Fig. 1C-E, the expression pattern of AKAP1 varies significantly among the HCC samples. The expression of AKAP1 was significantly increased in HCC samples relative to paired non-cancerous tissues in from $64.6 \%(102 / 158)$ of patients with HCC (Fig. 1F).

High AKAPl expression is associated with aggressive clinicopathological features. Based on IHC staining intensity and percentages of positive tumor cells (Fig. 2), the patients were subdivided into two groups: High $(n=107)$ and low $(n=51)$ AKAP1 expression groups. As depicted in Table I, AKAP1 
Table I. Association between AKAP1 expression and clinicopathological characteristics in 158 hepatocellular carcinoma patients.

\begin{tabular}{|c|c|c|c|c|}
\hline Variables & All cases & Low AKAP1 & High AKAP1 & P-value \\
\hline Total & 158 & 51 & 107 & \\
\hline Sex & & & & 0.414 \\
\hline Female & 17 & 4 & 13 & \\
\hline Male & 141 & 47 & 94 & \\
\hline Age, years & & & & 0.847 \\
\hline$<50$ & 73 & 23 & 50 & \\
\hline$\geq 50$ & 85 & 28 & 57 & \\
\hline HBV infection & & & & 0.944 \\
\hline Yes & 139 & 45 & 94 & \\
\hline No & 19 & 6 & 13 & \\
\hline Liver cirrhosis & & & & 0.420 \\
\hline Yes & 112 & 34 & 78 & \\
\hline No & 46 & 17 & 29 & \\
\hline AFP, ng/ml & & & & 0.828 \\
\hline$\geq 200$ & 91 & 30 & 61 & \\
\hline$<200$ & 67 & 21 & 46 & \\
\hline Tumor multiplicity & & & & 0.325 \\
\hline Single & 55 & 15 & 40 & \\
\hline Multiple & 103 & 36 & 67 & \\
\hline Tumor size, $\mathrm{cm}$ & & & & 0.024 \\
\hline$\leq 5$ & 52 & 23 & 29 & \\
\hline$>5$ & 106 & 28 & 78 & \\
\hline Tumor encapsulation & & & & 0.395 \\
\hline Absent & 79 & 23 & 56 & \\
\hline Present & 79 & 28 & 51 & \\
\hline Edmondson grade & & & & 0.903 \\
\hline $\mathrm{I} / \mathrm{II}$ & 35 & 11 & 24 & \\
\hline III/IV & 123 & 40 & 83 & \\
\hline Portal vein thrombosis & & & & 0.005 \\
\hline Absence & 117 & 45 & 72 & \\
\hline Gross & 41 & 6 & 35 & \\
\hline Pathologic TNM stage & & & & 0.030 \\
\hline Early stage (I-II) & 92 & 36 & 56 & \\
\hline Late stage (III) & 66 & 15 & 51 & \\
\hline
\end{tabular}

AKAP1, A-kinase anchoring protein 1; HBV, hepatitis B virus; AFP, $\alpha$-fetoprotein; TNM, Tumor-Node-Metastasis.

expression levels were significantly higher in $\mathrm{HCC}$ patients with increased tumor size $(\mathrm{P}=0.024)$, portal venous invasion $(\mathrm{P}=0.00498)$, and late TNM stage $(\mathrm{P}=0.0296)$. These data indicate that high AKAP1 expression is associated with aggressive clinicopathological features.

High AKAPl expression predicts poor overall survival $(O S)$ and disease-free survival (DFS) rates in a cohort of patients with $H C C$. To assess the association between AKAP1 expression levels with survival of patients with HCC, Kaplan-Meier survival analyses were performed. As depicted in Fig. 3, patients in the high expression group exhibited poorer DFS $(\mathrm{P}=0.002)$ and $\mathrm{OS}(\mathrm{P}=0.004)$ rates than those in the low expression group. In addition, to determine whether AKAP1 expression level is an independent prognostic factor for DFS rate and identify other prognostic factors for DFS rate in patients with HCC who underwent curative resection, Cox regression analysis was performed for 12 clinicopathological variables. Univariate analysis demonstrated that AKAP1 expression level was associated with DFS rate [hazard ratio (HR), 1.934; 95\% confidence interval (CI), 1.243-3.01; $\mathrm{P}=0.003$ ], and our multivariable Cox regression analyses further confirmed that AKAP1 expression levels were an independent risk factor of DFS rate (HR, 1.972; 95\% CI, 1.177-3.306; P=0.01) (Table II). These data indicated that high AKAP1 expression in tumors was 
Table II. Univariate and multivariate Cox regression analysis of risk factors for disease-free survival rate.

\begin{tabular}{|c|c|c|c|c|}
\hline \multirow[b]{2}{*}{ Variables } & \multicolumn{2}{|c|}{ Univariate analysis } & \multicolumn{2}{|c|}{ Multivariable analysis } \\
\hline & Hazard ratio $(95 \% \mathrm{CI})$ & $\mathrm{P}$-value & Hazard ratio $(95 \% \mathrm{CI})$ & P-value \\
\hline Sex (male vs. female) & $0.512(0.249-1.055)$ & 0.070 & & \\
\hline Age ( $\geq 50$ vs. $<50$ years $)$ & $0.876(0.595-1.289)$ & 0.501 & & \\
\hline HBV infection (present vs. absent.) & $1.067(0.585-1.948)$ & 0.832 & & \\
\hline Liver cirrhosis (present vs. absent) & $1.737(1.083-2.787)$ & 0.022 & $1.681(0.999-2.829)$ & 0.051 \\
\hline $\operatorname{AFP}(\geq 400 \mathrm{ng} / \mathrm{ml}$ vs. $<400 \mathrm{ng} / \mathrm{ml})$ & $1.245(0.842-1.841)$ & 0.272 & & \\
\hline Tumor multiplicity (multiple vs. single ) & $1.593(1.073-2.365)$ & 0.021 & $0.963(0.592-1.568)$ & 0.880 \\
\hline Maximal tumor size $(\geq 5$ vs. $<5 \mathrm{~cm})$ & $2.033(1.303-3.174)$ & 0.002 & $2.110(1.275-3.493)$ & 0.004 \\
\hline Tumor encapsulation (absent vs. present) & $1.707(1.111-2.621)$ & 0.015 & $1.381(0.865-2.206)$ & 0.176 \\
\hline Edmondson grade (III/IV vs. I/II) & $1.631(0.981-2.71)$ & 0.059 & & \\
\hline Portal vein thrombosis (gross vs. absence) & $2.329(1.543-3.516)$ & $<0.001$ & $1.461(0.892-2.395)$ & 0.132 \\
\hline Pathologic TNM stage (III vs. I-II) & $1.800(1.225-2.645)$ & 0.003 & $1.354(0.861-2.130)$ & 0.189 \\
\hline AKAP1 level (high vs. low) & $1.934(1.243-3.01)$ & 0.003 & $1.972(1.177-3.306)$ & 0.010 \\
\hline
\end{tabular}

AKAP1, A-kinase anchoring protein 1; HBV, hepatitis B virus; AFP, $\alpha$-fetoprotein; TNM, Tumor-Node-Metastasis; CI, confidence interval.

A

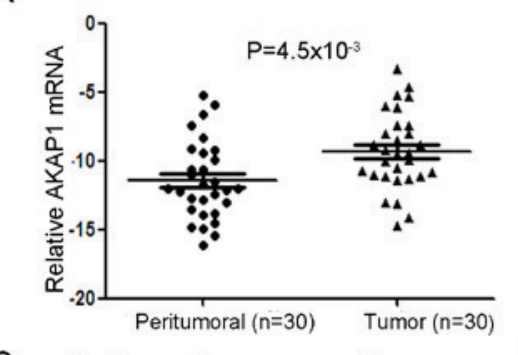

C

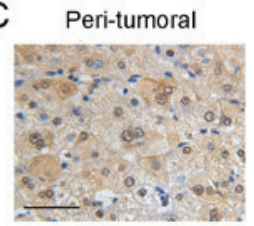

$\mathrm{E}$

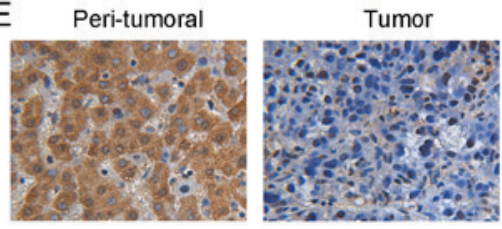

B

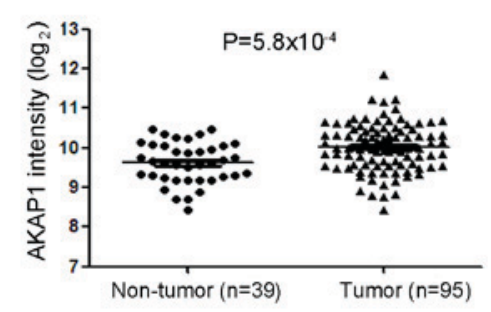

D Peri-tumoral Tumor
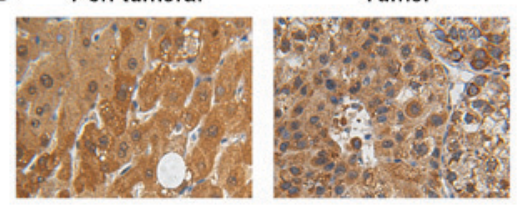

$\mathrm{F}$



Figure 1. AKAP1 is frequently upregulated in HCC tissues. (A) The mRNA expression of AKAP1 was examined by reverse transcription-quantitative polymerase chain reaction in 30 pairs of HCC tissues and matched peritumoral tissues. (B) AKAP1 expression was analyzed in HCC tumor and non-tumor tissues that were obtained from the Gene Expression Omnibus (accession number GSE45436). (C-E) Representative immunohistochemical staining of AKAP1 in tumor tissues and matched peritumoral tissues. (C) Upregulated (D) no change, and (E) downregulated. Scale bar, $50 \mu \mathrm{m}$. (F) The expression of AKAP1 was upregulated in $64.56 \%(102 / 158)$ of patients with HCC. AKAP1, A-kinase anchoring protein 1.

associated with disease recurrence and poor survival rates for patients with HCC, and AKAP1 could also be used as a valuable prognostic factor for the DFS rate of patients with HCC who underwent curative resection.

\section{Discussion}

Recently, evidence indicates that metabolic disorders have notable roles in HCC initiation, progression and therapy resistance. Obesity and diabetes have long been recognized as independent risk factors for the development of HCC (15-17).

As the energetic hub of the cell, mitochondria serve important roles in regulating cell function and survival $(18,19)$. Mitochondrial dysfunction is a common trait of several human diseases, including cancer (20-23). AKAP1 is a scaffolding protein that functions as the key regulatory molecule responsible for controlling mitochondria function. Therefore, it is 
A

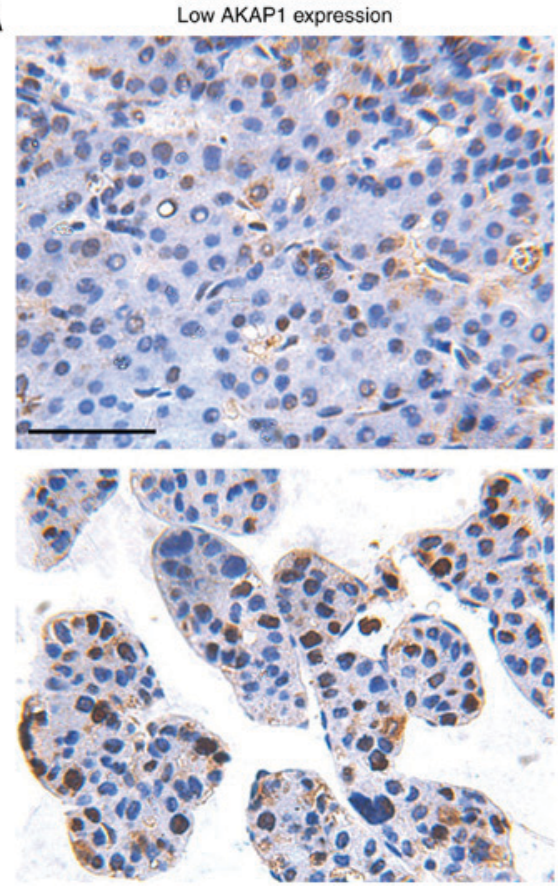

B
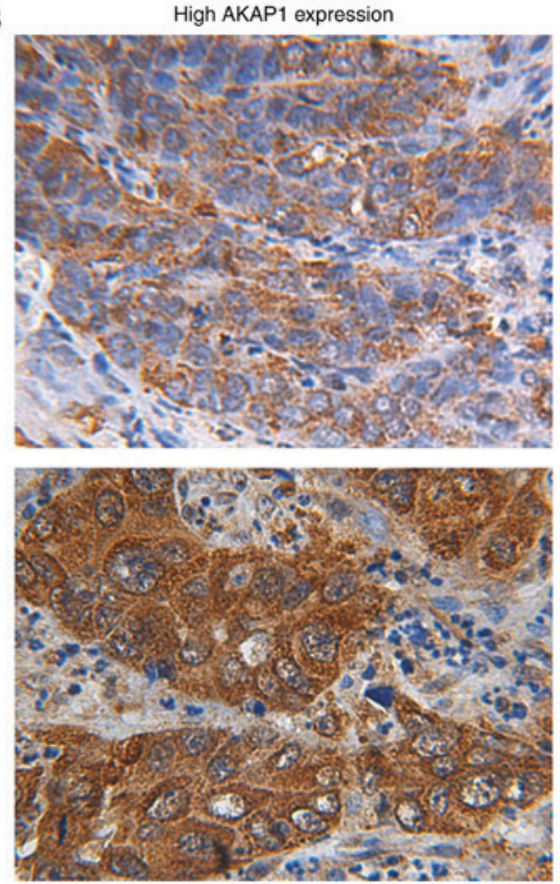

Figure 2. Representative immunostaining of AKAP1 in HCC. AKAP1 expression was evaluated by immunohistochemically staining. Representative (A) low and (B) high AKAP1 expression samples were shown. Scale bar, $50 \mu \mathrm{m}$. AKAP1, A-kinase anchoring protein 1.

A

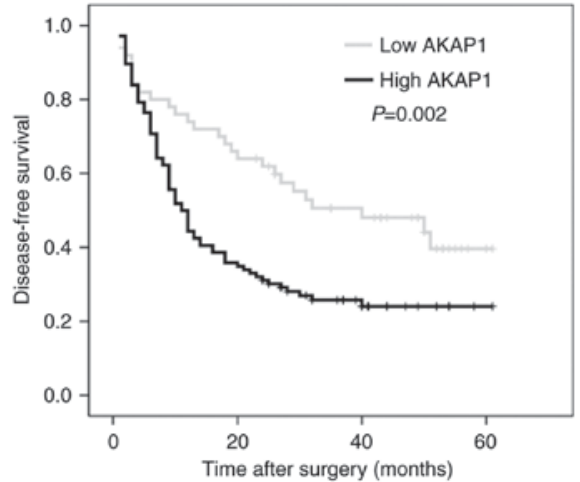

B

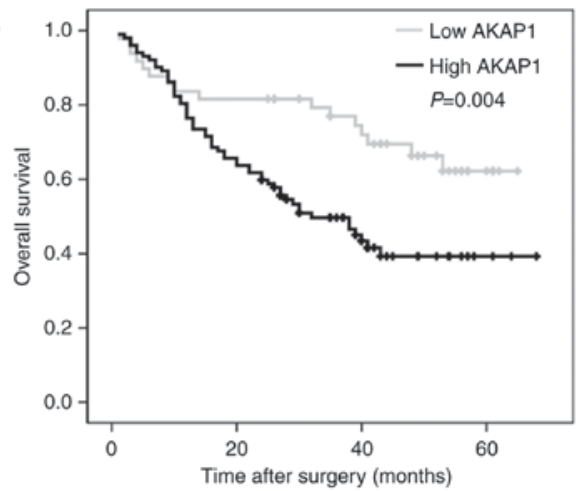

Figure 3. High expression of AKAP1 in tumors is associated with poor survival of patients with HCC. The (A) disease-free and (B) overall survival rates of 158 patients with HCC were compared between the low- and high-AKAP1 expression groups. AKAP1, A-kinase anchoring protein 1; HCC, hepatocellular carcinoma.

necessary to investigate the role of AKAP1 in cancer. In fact, the expression profiles and prognostic implications of AKAP1 have been documented in human non-small cell lung cancer, and AKAP1 overexpression is inversely associated with patient survival (13). The present study evaluated the expression level of AKAP1 in HCC for the first time. Through studying human HCC clinical specimens, it was revealed that AKAP1 was overexpressed in the majority of patients with $\mathrm{HCC}$, and high AKAP1 expression was associated with aggressive clinicopathological features. These data indicated that AKAP1 may contribute to progression of HCC.

Although early diagnosis and developments in surgery improved the short-term survival rates of patients with HCC (24), the long-term prognosis of patients with HCC remains unsatisfactory, even following radical excision. Therefore, it is necessary to reveal reliable biomarkers for the effective identification of patients with $\mathrm{HCC}$ who have a high risk of relapse following surgery. In the present study, Kaplan-Meier survival analyses revealed that high AKAP1 expression was associated with poor OS and DFS rates. Furthermore, univariate and multivariate survival analyses indicated that AKAP1 expression could serve as an independent factor for the DFS rate of patients with HCC following hepatectomy.

In conclusion, the results of the present study demonstrated that AKAP1 was overexpressed in the majority of patients with $\mathrm{HCC}$, and its increased expression was associated with poor patient prognosis. AKAP1 may serve as a valuable prognostic biomarker in predicting the survival of patients with HCC following radical resection.

\section{Acknowledgements}

Not applicable. 


\section{Funding}

This research was supported by the National Natural Science Foundation of China (grant no. 81000971).

\section{Availability of data and materials}

The datasets used and/or analyzed during the current study are available from the corresponding author on reasonable request.

\section{Authors' contributions}

JY and RL designed the experiments. JY, YZ, DXZ and JW performed the experiments, analyzed and interpreted the data, and wrote the manuscript. RL organized and supervised the study. All authors read and approved the final manuscript.

\section{Ethics approval and consent to publish}

All human sample collection procedures were approved by the Biomedical Ethics Committee of Eastern Hepatobiliary Surgery Hospital (Shanghai, China). Written informed consent for tissue banking and future medical research was obtained from each participant prior to surgery.

\section{Consent for publication}

Identifying information of patients were not included in the manuscript. Informed consent for tissue banking and future medical research was obtained from each participant prior to surgery.

\section{Competing interests}

The authors declare that they have no competing interests.

\section{References}

1. Ferlay J, Soerjomataram I, Dikshit R, Eser S, Mathers C, Rebelo M, Parkin DM, Forman D and Bray F: Cancer incidence and mortality worldwide: Sources, methods and major patterns in GLOBOCAN 2012. Int J Cancer 136: E359-E386, 2015.

2. Bertuccio P, Turati F, Carioli G, Rodriguez T, La Vecchia C, Malvezzi M and Negri E: Global trends and predictions in hepatocellular carcinoma mortality. J Hepatol 67: 302-309, 2017.

3. Torre LA, Bray F, Siegel RL, Ferlay J, Lortet-Tieulent J and Jemal A: Global cancer statistics, 2012. CA Cancer J Clin 65: 87-108, 2015.

4. Sharma SA, Kowgier M, Hansen BE, Brouwer WP, Maan R, Wong D, Shah H, Khalili K, Yim C, Heathcote EJ, et al: Toronto HCC risk index: A validated scoring system to predict 10 -year risk of HCC in patients with cirrhosis. J Hepatol: S0168-8278(17)32248-1, 2017.

5. Chen W, Zheng R, Baade PD, Zhang S, Zeng H, Bray F, Jemal A, Yu XQ and He J: Cancer statistics in China, 2015. CA Cancer J Clin 66: 115-132, 2016.

6. Fu J and Wang $\mathrm{H}$ : Precision diagnosis and treatment of liver cancer in China. Cancer Lett 412: 283-288, 2018.
7. Carlucci A, Adornetto A, Scorziello A, Viggiano D, Foca M, Cuomo O, Annunziato L, Gottesman $\mathrm{M}$ and Feliciello A: Proteolysis of AKAP121 regulates mitochondrial activity during cellular hypoxia and brain ischaemia. EMBO J 27: 1073-1084, 2008.

8. Czachor A, Failla A, Lockey R and Kolliputi N: Pivotal role of AKAP121 in mitochondrial physiology. Am J Physiol Cell Physiol 310: C625-C628, 2016.

9. Schiattarella GG, Cattaneo F, Pironti G, Magliulo F, Carotenuto G, Pirozzi M, Polishchuk R, Borzacchiello D, Paolillo R, Oliveti M, et al: Akap1 deficiency promotes mitochondrial aberrations and exacerbates cardiac injury following permanent coronary ligation via enhanced mitophagy and apoptosis. PLoS One 11: e0154076, 2016.

10. Smith FD, Langeberg LK and Scott JD: The where's and when's of kinase anchoring. Trends Biochem Sci 31: 316-323, 2006.

11. Cardone L, Carlucci A, Affaitati A, Livigni A, DeCristofaro T, Garbi C, Varrone S, Ullrich A, Gottesman ME, Avvedimento EV and Feliciello A: Mitochondrial AKAP121 binds and targets protein tyrosine phosphatase D1, a novel positive regulator of src signaling. Mol Cell Biol 24: 4613-4626, 2004.

12. Rinaldi L, Sepe M, Delle Donne R, Conte K, Arcella A, Borzacchiello D, Amente S, De Vita F, Porpora M, Garbi C, et al: Mitochondrial AKAP1 supports mTOR pathway and tumor growth. Cell Death Dis 8: e2842, 2017.

13. Bruix J and Sherman M; Practice Guidelines Committee, American Association for the Study of Liver Diseases: Management of hepatocellular carcinoma. Hepatology 42: 1208-1236, 2005.

14. Livak KJ and Schmittgen TD: Analysis of relative gene expression data using real-time quantitative PCR and the 2(-Delta Delta C(T)) method. Methods 25: 402-408, 2001.

15. Starley BQ, Calcagno CJ and Harrison SA: Nonalcoholic fatty liver disease and hepatocellular carcinoma: A weighty connection. Hepatology 51: 1820-1832, 2010.

16. El-Serag HB, Tran T and Everhart JE: Diabetes increases the risk of chronic liver disease and hepatocellular carcinoma. Gastroenterology 126: 460-468, 2004.

17. Yang JD, Mohamed HA, Cvinar JL, Gores GJ, Roberts LR and Kim WR: Diabetes mellitus heightens the risk of hepatocellular carcinoma except in patients with hepatitis C cirrhosis. Am J Gastroenterol 111: 1573-1580, 2016.

18. Henze K and Martin W: Evolutionary biology: Essence of mitochondria. Nature 426: 127-128, 2003.

19. Weinberg SE and Chandel NS: Targeting mitochondria metabolism for cancer therapy. Nat Chem Biol 11: 9-15, 2015.

20. Vazquez F, Lim JH, Chim H, Bhalla K, Girnun G, Pierce K, Clish CB, Granter SR, Widlund HR, Spiegelman BM and Puigserver P: PGClalpha expression defines a subset of human melanoma tumors with increased mitochondrial capacity and resistance to oxidative stress. Cancer Cell 23: 287-301, 2013.

21. Genini D, Brambilla L, Laurini E, Merulla J, Civenni G, Pandit S, D'Antuono R, Perez L, Levy DE, Pricl S, et al: Mitochondrial dysfunction induced by a SH2 domain-targeting STAT3 inhibitor leads to metabolic synthetic lethality in cancer cells. Proc Natl Acad Sci USA 114: E4924-E4933, 2017.

22. Yuan D, Huang S, Berger E, Liu L, Gross N, Heinzmann F, Ringelhan M, Connor TO, Stadler M, Meister M, et al: Kupffer cell-derived Tnf triggers cholangiocellular tumorigenesis through JNK due to chronic mitochondrial dysfunction and ROS. Cancer Cell 31: 771-789.e6, 2017.

23. Viale A, Pettazzoni P, Lyssiotis CA, Ying H, Sánchez N, Marchesini M, Carugo A, Green T, Seth S, Giuliani V, et al: Oncogene ablation-resistant pancreatic cancer cells depend on mitochondrial function. Nature 514: 628-632, 2014.

24. Bruix J, Reig M and Sherman M: Evidence-based diagnosis, staging, and treatment of patients with hepatocellular carcinoma. Gastroenterology 150: 835-853, 2016.

(i) (5) () This work is licensed under a Creative Commons cc) $\mathrm{EY}$ No ND Attribution-NonCommercial-NoDerivatives 4.0 International (CC BY-NC-ND 4.0) License. 